\section{$\underset{\substack{\text { hommes } \\ \text { \& migrations }}}{ }$}

\section{Hommes \& migrations}

Revue française de référence sur les dynamiques

migratoires

$1312 \mid 2015$

Diasporas iraniennes

\title{
D’une génération à l'autre, transmettre le « capital communautaire »
}

Quels héritages pour les jeunesses d'origine turque?

\section{Maïtena Armagnague}

\section{(2) OpenEdition}

Journals

Édition électronique

URL : http://journals.openedition.org/hommesmigrations/3513

DOI : $10.4000 /$ hommesmigrations.3513

ISSN : 2262-3353

Éditeur

Musée national de l'histoire de l'immigration

Édition imprimée

Date de publication : 1 octobre 2015

Pagination : 120-124

ISBN : 978-2-919040-33-9

ISSN : 1142-852X

Référence électronique

Maïtena Armagnague, "D'une génération à l'autre, transmettre le "capital communautaire » »,

Hommes \& migrations [En ligne], 1312 | 2015, mis en ligne le 31 mai 2016, consulté le 15 septembre 2020. URL : http://journals.openedition.org/hommesmigrations/3513 


\section{D'UNE GÉNÉRATION À L'AUTRE, TRANSMETTRE LE "CAPITAL COMMUNAUTAIRE" QUELS HÉRITAGES POUR LES JEUNESSES D'ORIGINE TURQUE ?}

par MAÏTENA ARMAGNAGUE, maître de conférences en sociologie à INS HEA/université Paris-Lumières (UPL), chercheure au GRHAPES, associée au Centre Émile-Durkheim.

C inquante ans après la signature du contrat bilatéral d'immigration entre la France et la Turquie, on observe que l'évolution des migrations turques n'est pas homogène. Pour les descendants des migrants, il n'existe pas "une seule" façon de prendre part à la société française, contrairement aux résultats des premières enquêtes démographiques'. Les Turcs et leurs descendants n'ont pas tous connu les mêmes parcours sociaux en France ${ }^{2}$, selon leur région et leur position sociopolitique en Turquie (niveau d'instruction, origine rurale ou urbaine, orientation politique à droite ou à gauche) et leur parcours d'immigration en France (implantation en zone urbaine ou rurale, en zone industrialisée ou non). Dans chaque cas, des "accommodations" ont eu lieu dans des conjonctures socio-économiques et urbaines locales.

La France est le second pays d'immigration des Turcs après l'Allemagne. La présence de jeunes générations témoigne de l'installation de cette immigration dans la durée. Cette jeunesse s'illustre aujourd'hui par son hétérogénéité, construite à la faveur de différentes transmissions générationnelles et de la façon dont les patrimoines ont pu, ou non, être activés dans des espaces locaux dépendants de conjonctures socio-économiques et urbaines spécifiques 3 .

\section{Activation conjoncturelle des héritages communautaires}

Les entrées de Turcs en France, nombreuses à partir du milieu des années $1970^{4}$, se font d'abord dans les villes de l'est de la France (I'“axe turc" rhénan), en île-de-France et en Rhône-Alpes, puis progressivement vers le centre et l'ouest du pays, notamment dans des zones moins urbaines ${ }^{5}$ ou historiquement moins industrialisées (Limousin, Normandie, Bretagne, Gironde). Ainsi, les migrants turcs s'installent sur la façade atlantique ${ }^{6}$, prin-

1. Emmanuel Todd, Le Destin des immigrés. Assimilation et ségrégation dans les démocraties occidentales, Paris, Seuil, 1994 ; Michèle Tribalat, Faire France. Une enquête sur les immigrés et leurs enfants, Paris, La Découverte, 1995 ; Michèle Tribala, De l'immigration à l'assimilation. Enquête sur les populations d'origine étrangère en France, Paris, La Découverte/INED, 1996. 2. Maïtena Armagnague, "Les dynamiques d'adaptation sociale des communautés turques en France et en Allemagne. Le cas des jeunes générations", in Sociologie, $n^{\circ} 2$, vol. 1, 2010. 3. Maïtena Armagnague, Une jeunesse turque en France et en Allemagne, Lormont, éd. du Bord de l'eau, 2016. 4. Isabelle Rigoni, "Les Turcs d'Europe : bilan d'une migration", in Migrations Société, vol. 17, $n^{\circ}$ 98, 2005 ; Isabelle Rigon, "Actions, mobilisations et recompositions chez les migrants de Turquie", Paris, thèse de doctorat en sciences politiques, 2000. 5. Gülsen Yildirim, "Les Turcs en milieu rural. Le cas du Limousin", in Gaye Petek (dir.),

"Les Turcs en France. Quels ancrages?", Hommes \& Migrations, n 1280, 2009. 6. Notre recherche menée en France porte plus particulièrement sur la Gironde. Voir Une jeunesse turque en France, op. cit. 
cipalement à partir du début des années 1980, dans un contexte politique en Turquie sensible?. En France, cette période correspond à la fin des Trente Glorieuses et du quasi-plein emploi qui était à l'origine de nombreuses entrées migratoires. Du fait de cette situation, certains migrants turcs, entrés tardivement et devenus artisans, n'accèdent pas au salariat que permettait la prospérité d'aprèsguerre $^{8}$. Ces Turcs ont alors privilégié des modalités plus "communautaires" de participation économique, du fait notamment de leurs ressources diasporiques ${ }^{9}$. En conséquence, ils ont moins directement subi le ralentissement de l'économie française, protégés dans des "niches" économiques $^{10}$. De plus, certains salariés se sont orientés vers des entreprises turques après avoir perdu leur emploi d'ouvrier du fait du ralentissement économique. Cette situation a contribué à produire des mécanismes de distinction symbolique vis-àvis des autres populations migrantes et musulmanes originaires d'Afrique"1, qui connaissent le chômage et la marginalisation et auxquelles les Turcs étaient souvent associés par les institutions, notamment éducatives ${ }^{12}$. Cette communauté est

7. Hamit Bozarslan, Histoire de la Turquie. De l'Empire à nos jours, Paris, Tallandier, 2013. 8. À Bordeaux, par exemple, la faiblesse relative de l'emploi industriel rend cette situation particulièrement saillante mais il nous faut toutefois nuancer cette observation : sur la rive droite de l'agglomération, des ressortissants turcs, parmi les premiers à avoir immigré, ont été recrutés par l'usine automobile Ford de Blanquefort, qui a failli fermer ses portes en 2010 et fonctionne, depuis, au ralenti. 9. Stéphane de Tapia, "Dimensions transnationales et culturelles des migrations turques en Europe", in Multitudes, n 49, vol. 2, 2012. 10. Mark Granovetter, "La sociologie économique des entreprises et des entrepreneurs", in Terrains et travaux, n 4, 2003, pp. 167-206; Roger Waldinger, Howard Aldrich, Robin Ward et al., Ethnic Entrepreneurs. Immigrant Business in Industrial Societies, Newbury, CA, Sage

Publications, 1990. 11. Riva Kastoryano, Être turc en France. Réflexions sur familles et communauté, Paris, L’Harmattan, 1986.

12. Maïtena Armagnague, "Le village contre le ghetto. La dimension ethnique dans la cité, une ressource pour les descendants de migrants", in Régis Cortéséro (dir.), La Banlieue change! Inégalités, justice sociale et action publique dans les quartiers populaires, Lormont, éd. du Bord de l'eau, 2012, pp.115-129. 


\section{MÉMOIRES}

donc une construction sociale dépendante d'un contexte ${ }^{13}$. Elle s'est, ou ne s'est pas, construite selon les lieux. Elle a également évolué de façon variable selon les socialisations transmises d'une génération à l'autre. Ainsi, on peut parler de différentes jeunesses d'ascendance turque, qui ne se résument pas à des productions sociales purement collectives, exclusivement déterminées par des ressources transnationales diasporiques ou par un "modèle" politique et économique national. Ces jeunesses ne se réduisent pas non plus à des expériences strictement individuelles, comme le suggèrent parfois les figures de réussite érigées en "exemples". Ainsi, pour comprendre les jeunes descendants des migrations turques, il n'est pas suffisant de comprendre les Turcs. II faut aussi prendre en compte la société française et ses institutions de socialisation, comme le sort que la France réserve tant à ses migrants et descendants, qu'à sa jeunesse en général.

\section{"Faire communauté" : une transmission fonctionnelle}

Au-delà de l'éventuelle conformité aux espaces communautaires turcs ou aux normes majoritaires, la famille peut être porteuse d'aspirations à la mobilité sociale, notamment dans les milieux populaires ${ }^{14}$. Ainsi, les familles composent en permanence leur adhésion à la communauté, comme elles composent leur rapport aux institutions fran- çaises ${ }^{15}$, notamment en fonction du parcours scolaire de leur progéniture. C'est aussi en fonction de l'expérience scolaire que se fera, plus ou moins, la transmission de l'héritage et des capitaux culturels turcs (appartenances et pratiques culturelles, politiques et religieuse) ou, au contraire, leur mise en retrait par la famille (qui s'éloigne parfois des lieux communautaires ou n'incite pas les enfants à s'y engager). Soulignons à cet égard la grande hétérogénéité politique et socioculturelle de l'immigration turque ${ }^{16}$ et donc des transmissions potentielles des aînés. Les jeunes n'ont pas tous reçu et entretenu le même rapport à la Turquie et à son histoire, du fait des différences sociales et politiques de leurs parents ou grands-parents avant la migration et du fait des trajectoires différentes après la migration. Le rapport des migrants turcs et de leurs descendants à l'histoire nationale turque ou à la religion peut aussi être influencé par leur affiliation à différentes organisations transnationales - associations turques ou kurdes, alévies ou sunnites : mouvement Milli Görüs, confréries Suleymancilar, Nurculuk, mouvance de Güllen - qui vont participer à construire des positionnements sociaux différents ${ }^{17}$.

Les aînés, relativement préservés du chômage, ont souvent transmis à leurs enfants ces héritages culturels et religieux, en privilégiant différemment certaines valeurs (laïcité et/ou nationalisme et/ou appartenance religieuse) selon l'organisation politique et/ou religieuse dont ils se sentaient proches. Ils le faisaient par attachement idéolo-

13. Klaus Lichtblau, “Vergemeinschaftung' und 'Vergesellschaftung' bei Max Weber. Eine Rekonstruktion seines Sprachgebrauchs”, in Zeitschrift für Soziologie, Jg. 29, Heft 6, Dezember 2000, pp. 423-443; Max Weber, Économie et société, Paris, Agora Pocket, 2003 [1922]. 14. Emmanuelle Santelli, La Mobilité sociale dans l'immigration. Itinéraire de réussite des enfants d'origine algérienne, Toulouse, Presses universitaires du Mirail, 2001; Bernard Lahire, Tableaux de familles. Heurs et malheurs scolaires en milieux populaires, Paris, Seuil, 1995. L'ouvrage d'Emmanuelle Santelli portant sur des parcours de "réussite sociale" montre l'influence de la famille, ou de "référents éducatifs" (tels des voisins) en mesure de soutenir la scolarité, sur les performances scolaires. Dans les milieux populaires, l'ouvrage de Bernard Lahire souligne que les choix éducatifs et les configurations familiales construites notamment par rapport à l'école peuvent être décisifs sur les résultats scolaires comme, par exemple, le fait de pouvoir disposer d'un lieu privé pour étudier le soir ou d'un aîné en mesure de restituer son propre parcours, ce qui permet d'identifier et de mieux surmonter les difficultés scolaires. 15. Mathieu Ichou, Marco Oberti, "Le rapport à l'école des familles déclarant une origine immigrée : enquête dans quatre lycées de la banlieue populaire," in Population, forthcoming, 2014. 16. Antoine Pécoud, "Réseaux, ethnicité et institutions dans les économies immigrées", in Hommes \& Migrations, n 1250, 2004, pp. 13-23. 17. Samim Akgönül, Religions de Turquie, religions des Turcs. Nouveaux acteurs dans l'Europe élargie, Paris, L'Harmattan, 2006. 
gique et aussi pour maintenir les réseaux sociaux familiaux et de voisinage offrant des socialisations professionnelles et extraprofessionnelles, car les réseaux économiques sont souvent télescopés aux associations politiques et/ou religieuses ${ }^{18}$ : recrutement entre compatriotes et enfants de compatriotes dans les entreprises turques, loisirs, culte, organisation des alliances et des obsèques. Toutefois, ces ressources peuvent devenir inutiles dès lors qu'une réussite scolaire offre l'opportunité de s'inscrire individuellement dans la société.

Observons que la transmission des capitaux communautaires ne se fait pas de manière "descendante", comme un simple patrimoine légué : elle est construite conjointement et réciproquement par les différentes générations en présence. Ainsi, les ressources communautaires (réseaux associatifs ou liens à des entreprises turques) sont entretenues par une partie de la jeunesse d'origine turque qui va construire des parcours sociaux (choix matrimoniaux, maîtrise de la langue turque, investissement dans les associations et parfois emploi dans les entreprises turques). Dans ces cas, les liens communautaires sont constitués en capitaux mobilisables; ils ne sont ni une "première ressource" du processus migratoire, ni une seule réaction au racisme, aux discriminations et aux relégations subies. Ces liens sociaux constituent, pour ces jeunes, un ordre social quotidien et ordinaire, qu'ils questionnent peu, notamment dans les fréquentations ou les alliances. Pour autant, cet ordre social est négocié, notamment par les filles ${ }^{19}$. Lorsque des jeunes, notamment des filles, ont des performances scolaires assez élevées pour leur permettre d'accéder à des filières sélectives offrant des débouchés professionnels opératoires, ils s'éloignent de la communautéz ${ }^{\circ}$, du fait d'un parcours scolaire individualisé (changement d'établissement pour aller en filière Sciences Po, mobi- lité géographique pour les études supérieures). Mais de tels parcours sont relativement limités. Contrairement à ce que croient les acteurs scolaires ou d'autres acteurs institutionnels (élus, responsables associatifs ou de lieux d'intervention sociale), la "réussite" sociale via l'école ne peut, dans une conjoncture économique tendue, remettre en cause les liens communautaires et leur transmission. La réussite individuelle, conforme aux valeurs du modèle républicain, ne brise pas la communauté. C'est la marginalisation sociale spatiale et générationnelle qui fragilise cette communauté durablement ${ }^{21}$.

\section{La minorité contre la communauté}

La communauté, au sens d'un ensemble de liens sociaux solidaires, est menacée par un processus de marginalisation d'une partie des jeunes d'ascendance turque qui s'incorpore aux jeunesses marginalisées des quartiers urbains défavorisés. Les liens aux espaces communautaires se perdent et ces jeunes ne peuvent plus compter sur les ressources ethniques des entreprises turques. Paradoxalement, c'est l'école qui crée et organise les conditions de cette mobilité descendante en regroupant en son sein diverses jeunesses issues des zones urbaines reléguées. Ainsi, la communauté traditionnelle turque perd de son influence au profit d'une socialisation juvénile fédérant un groupe d'âge du quartier. Ce collectif, construit symboliquement comme une minorité, ne se définit pas grâce à ce qui a été transmis par les aînés, en termes de valeurs et de liens sociaux, mais par les expériences communes avec un environnement perçu comme dominant. Cette expérience s'appuie sur une vision plus duale des rapports

18. André Levallois, "De la migration à l'insertion, Les Turcs en Alsace”, in Hommes \& Migrations, $n^{\circ} 1153,1992, p p .10-12$. 19. Feyza Ak Akyol "Construction de l'identité féminine turque dans le processus migratoire. Le cas Turquie-France”, thèse de doctorat, université Aix-Marseille-I, 2005. 20. L'éloignement de la communauté peut prendre des formes différentes (surinvestissement scolaire, négociation pour ne pas aller à tous les rassemblements communautaires; dont religieux) et repose notamment sur l'école. Certaines formes plus radicales peuvent conduire des jeunes filles à la fugue. 21. Maïtena Armagnague, Une jeunesse turque en France et en Allemagne, op. cit. 


\section{MÉMOIRES}

sociaux de classe, générationnels et ethniques. Les liens communautaires qui faisaient office d'espaces intermédiaires ne sont alors plus opérants ${ }^{22}$ Mais, d'un autre côté, les scolarités heurtées de ces jeunes leur ferment les portes d'une mobilité sociale plus "classique". Les jeunes connaissent alors un repli ${ }^{23}$ et sont animés par le ressentiment à l'égard des institutions éducatives ou sociales. Dans certains cas, s'ils ont été en contact avec ces associations, ils adhèrent aux positions des organisations turques les plus ultra-nationalistes et les plus intégristes en convertissant certains héritages migratoires en force politique et religieuse d'opposition radicale ${ }^{24}$. Ce radicalisme politique et religieux ne vient pas de la tradition culturelle de la communauté, il puise ses sources dans des organisations turques transnationales et dans le monde politique turc lui-même ${ }^{25}$. II ne découle pas des mécanismes migratoires eux-mêmes, il procède d'une scène nationale et internationale, et une partie des jeunes y trouvent un sens, une raison d'agir.

Si ces organisations avaient peu d'audience chez les parents, force est de constater que leurs enfants $y$ adhèrent quelquefois ${ }^{26}$, selon des vertus morales concurrentielles à celles des parents qui potentiellement ne connaissent pas de limite. L'adhésion à cette minorité laisse peu de marge de liberté car elle exerce un contrôle social fort sur les jeunes. Ainsi la minorité, comme expérience, s'oppose à la communauté, comme ensemble de liens solidaires. Elle ne constitue pas pour autant une alternative socialisante vers la société majoritaire, mais une force d'opposition et de contestation amère.

Cette distinction entre communauté et minorité permet d'identifier des facteurs de risque différents parmi les "groupes" des jeunesses urbaines issues des migrations, généralement associés indifféremment à une altérité. Elle permet une lecture plus précise du monde social des migrants turcs et de leurs descendants. Elle révèle que les expériences de jeunesse dans l'immigration ne sont pas qu'affaire de transmissions patrimoniales, quand bien même celles-ci seraient recomposées et subjectivées. Ces expériences juvéniles sont des expressions politiques, même si les représentants des institutions majoritaires ne parviennent pas toujours à leur donner un sens ${ }^{27}$. Ainsi, la condition des migrants et de leurs descendants se comprend à travers l'analyse de la place faite aujourd'hui aux jeunes des milieux populaires, interrogeant les mécanismes scolaires généraux, les conditions d'accès à l'emploi et les manières d'habiter les villes et les quartiers.

\footnotetext{
22. Alejandro Portes, Rubén G. Rumbaut, Immigrant America. A Portrait, Berkeley (CA), University of California Press, 2006. 23. Herbert J. Gans, "Seconde generation decline. Scenarios for economics and ethnic futures of the post-1965 American immigrants", in Ethnic and Racial Studies, vol. 15, n², 1992, pp. 173-192. 24. C'est le cas notamment de jeunesses urbaines marginalisées qui reconstruisent une "culture de la rue" à partir de ces héritages turcs ultra-nationalistes ou construisent une ethnicité urbaine à partir du fondamentalisme religieux. Voir Maïtena Armagnague, Une jeunesse turque en France et en Allemagne, op. cit. 25. Altaï Manço, “Turcs d’Europe : de l'image tronquée à la complexité d'une réalité sociale immigrée", in Hommes \& Migrations, n 1226, 2000, pp. 76-87. 26. Ceci participe à la production d’une ethnicité juvénile urbaine caractérisant les jeunesses d’origine turque marginalisées. 27. Michel Kokoreff, La Force des quartiers. De la délinquance à l'engagement politique, Paris, Payot, 2003.
} 


\section{MÉMOIRES}

\section{LES ÉLUS FRANÇAIS D'ORIGINE TURQUE}

par ESER DURMAZ MARTINS, étudiante en master en sociologie et anthropologie (MIRI),

Université Paris-VI-Denis-Diderot.

E n mai 2015 la communauté turque fêtait ses cinquante ans d'immigration en France. Cette migration économique, tournée d'abord vers l'Allemagne, est venue s'installer en Alsace, en île-deFrance et en Rhône-Alpes, entre les années 1960 et le début des années 1980. Les résultats d'une petite enquête sur les élus municipaux d'origine turque permettent de mesurer le degré d'intégration de cette communauté dans la société française.

À la demande de la revue Hommes \& Migrations, une enquête' ${ }^{1}$ a été réalisée, entre décembre 2015 et début janvier 2016, auprès d'une cinquantaine d'élus d'origine turque exerçant actuellement un mandat municipal. S'appuyant sur un questionnaire dont le contenu a été défini en collaboration avec la sociologue Gaye Petek, cette enquête interroge les élus sur leur histoire familiale, les conditions de leur arrivée en France et d'autres dimensions socio-économiques qui expliquent leur accession à une responsabilité politique $^{2}$. Ce premier état de leurs parcours et expériences permet de comprendre qui sont ces "élus interculturels".

\section{Profils et identités socioculturelles}

Tout d'abord, les élus d'origine turque, dont l'âge oscille entre 37 et 55 ans, résident principalement dans la ville ou la région dans laquelle ils sont arrivés, voire où ils sont nés, ce qui confirme leur enracinement local. Comme évoqué plus haut, ces élus sont majoritairement installés dans les trois régions à forte implantation industrielle qui, à l'époque, étaient à la recherche d'une maind'œuvre peu qualifiée. C'est donc là que leurs pères sont venus pour occuper des poste d'ouvriers. Ces derniers sont arrivés seuls et ont été rejoints quelques années plus tard par leurs femmes et enfants dans le cadre du regroupement familial. Cependant, un tiers des élus interrogés sont nés en France. On note aussi qu'une élue a migré après s'être mariée en Turquie, et qu'un autre, venu pour des études universitaires, est finalement resté en France. Tous ont grandi dans des quartiers habités par des populations immigrées et sont parvenus à faire des études supérieures.

Pour ce qui est de leur statut marital, deux élus seulement sont célibataires, dont un vit en concubinage, et les neuf autres sont mariés. L'endogamie, très fréquente dans la communauté turque, se retrouve chez ces élus. En effet, seul un élu a pour conjointe une Française, tous les autres étant mariés soit à des membres de leur communauté d'origine, soit à des citoyennes turques. Les élus mariés ont en moyenne 3 enfants, ce qui les situe au-dessus de la moyenne française. Ils parlent turc avec leur conjoint(e). Ils déclarent parler également turc avec leurs parents mais utiliser le francais avec leurs enfants et leurs frères et sœurs. Culturellement, si la plupart d'entre eux se définissent comme Franco-Turcs en ajoutant presque systématiquement qu'ils se sentent plus Français 
que Turcs, deux d'entre eux précisent qu'ils sont "musulmans". Ils se déclarent appartenir aux deux cultures. L'un d'entre eux ajoute: "Je suis d'origine turque, très attachée, à ma culture d'origine, mais je me sens français avant tout. J'aime la France et ses valeurs des droits de l'homme. Certaines personnes voudraient nous cloisonner, nous étiqueter, mais il ne faut pas tomber dans ce piège."

\section{De l'association à la politique locale}

Dans les communes ou les départements qui ont accueilli les immigrés turcs en nombre, les communautés turques ont eu tendance à s'enraciner. Leur poids démographique a favorisé l'émergence d'une vie associative très riche que ces élus citent comme un véritable tremplin vers la vie politique. Selon le scénario le plus fréquent, c'est leur participation active à la vie associative du quartier ou de leur ville qui a permis à ces futurs élus d'être remarqués par les candidats aux élections municipales et d'être invités à les rejoindre sur leur liste. Les difficultés auxquelles les élus sont le plus confrontés suite à leur engagement se trouvent du côté familial, quand l'étiquette politique n'est pas conforme à celle de leurs parents. Un élu précise : "Le plus difficile pour un enfant d'immigré, c'est de faire comprendre à son papa que l'on s'engage politiquement mais avec la droite." D'autre part, d'autres candidats associent leur présence sur la liste municipale à des "parachutages" qui les ont obligés à devoir constamment légitimer leur choix et leur engagement auprès des autres élus. Un seul élu d'origine turque a réellement eu le sentiment d'avoir été recruté localement comme un candidat "prétexte". Il précise : "Quand j'ai commencé à participer aux réunions, j'ai vite vu que nous étions de simples pions, on était là juste pour décorer la salle. Les élus n'avaient pas du tout l'intention de nous écouter ou de nous donner des délégations. Ce que bien entendu j'ai dénoncé, et j'ai claqué la porte pour défendre les intérêts de ma ville."

\section{Rôle et vision politiques}

On constate au sujet des élus ayant un parcours lié à leur engagement dans la vie associative que leur couleur politique varie en fonction de l'opportunité qui leur a été donnée. Ce n'est pas le résultat 
d'un militantisme local. II y a autant d'élus engagés à droite qu'à gauche, ce qui peut surprendre. En tant qu'élue, quatre personnes interrogées présentent leur rôle comme celui d'un ambassadeur de la communauté turque auprès de la mairie, sans oublier de préciser qu'elles sont également au service de tous les citoyens. Un élu témoigne : "Je suis élu pour être l'ambassadeur de la communauté turque avec la mairie et les autres instances mais aussi l'ambassadeur de toute la diversité. Je suis élu pour servir les habitants de ma ville en général." Même si ces élus jouent un rôle de médiation avec leur communauté d'origine, officiellement ils occupent tous des responsabilités plus classiques comme adjoint au commerce, à la petite enfance ou à l'urbanisme... et assument leurs responsabilités sur ces questions.

Concernant la politique française de l'immigration, ils considèrent unanimement que les efforts devraient se concentrer sur l'intégration et non pas sur l'assimilation des immigrés. Beaucoup d'entre eux pensent que les événements tragiques du mois de novembre dernier ont un lien avec l'échec de cette politique. Un élu souligne : "I $y$ a des approches différentes qu'on peut faire surce sujet. À une certaine époque, la France ou plutôt les pouvoirs successifs en place ont laissé de côté certains citoyens et s'en sont très peu occupés. Cette attitude a créé une rupture entre ces communautés d'origine immigrée et le reste de la population. Aujourd'hui, nous en vivons la conséquence néfaste. Le résultat de cette rupture, c'est le développement d'une sorte de communautarisme que l'on n'arrive pas à maîtriser. Le vide créé par les pouvoirs publics est récupéré par le communautarisme religieux qui, à mon sens, est très dangereux pour la République. Les événements dramatiques que nous avons vécus en 2015 en sont des exemples parlants." Un élément fondamental que l'on retrouve-dans les discours de ces élus d'origine turque est que la diversité de la population française doit être respectée tout en luttant contre le communautarisme qui risque d'exclure certains groupes de la société. On peut être français tout en conservant ses spécificités culturelles.

\section{Devenir un élu tout court}

Lorsque l'on analyse le taux de réponses à cette enquête et la nature des échanges avec les élus d'origine turque, une forme de défiance (malgré la garantie de l'anonymat) s'est notamment exprimée chez les femmes interrogées par un très faible taux de participation et, chez les hommes, par le fait que deux élus aient refusé de répondre par écrit ou par téléphone et accepté uniquement l'entretien en tête à tête. Depuis l'arrivée et l'installation de la communauté turque en France, ces élus estiment qu'un grand chemin a été parcouru dans l'intégration des immigrés d'origine turque, comme en témoignent leurs parcours. Ils insistent aussi sur le fait que le communautarisme est moins présent chez les jeunes générations. Un élu souligne: "Nous avons une jeunesse très bien intégrée et on constate son évolution en termes de carrière professionnelle et d'implication dans la politique depuis quelques années." Un autre précise malgré tout : "Notre communauté s'est développée autour des valeurs du matérialisme. On veut tout, tout de suite. Cet argent ne leur sert même pas en France, ils bâtissent des maisons à 4000 kilomètres d'ici, alors qu'ils ne voient pas la destruction spirituelle de leurs enfants devant leur nez sous leur toit." Un des points marquants de l'enquête est que leur engagement politique résulte d'une sollicitation extérieure et moins d'une démarche personnelle, ce qui peut expliquer pourquoi ils sont surtout considérés aujourd'hui par les élus locaux comme les représentants d'une certaine communauté d'électeurs. La question qui reste en suspens est le nombre d'années qu'il faudra attendre pour voir des élus d'origine turque ailleurs que dans les villes où est ancrée leur communauté d'origine. 